\title{
Macular recovery function (nyctometry) in diabetics without and with early retinopathy
}

\author{
E Midena, T Segato, $M$ Giuliano, $M$ Zucchetto
}

\begin{abstract}
Nyctometry was used to assess macular recovery function in 234 diabetic patients; their retinopathy ranged from no retinopathy (99) to early background retinopathy (135). None had visual symptoms or macular oedema. Abnormal (reduced) nyctometry findings were significantly and directly related to the deterioration of diabetic retinopathy. There was no significant association between reduced or normal nyctometry findings and glycaemia at the time of the examination. The value of nyctometry in screening and follow-up is discussed.
\end{abstract}

Diabetic retinopathy is considered to be a disease affecting either retinal blood vessels or neurosensory retina. ${ }^{\prime}$ The neurosensory alterations are caused in part by vascular alterations, but they also, and perhaps primarily, reflect the local and systemic metabolic derangements due to diabetes. The retinal neurosensory losses may predict the evolution of retinopathy toward more advanced forms ${ }^{2-4}$ and sometimes may precede the onset of clinically detectable retinopathy. ${ }^{5-7}$ Such tests therefore seem to be useful not only for predicting the evolution of established diabetic retinopathy, but also for monitoring the retinal state of diabetic patients, when better control or new drugs are given to prevent the appearance of clinical retinopathy. The neurosensory losses involve macular recovery function among others.

The present study was undertaken to examine in more detail the relationship between modifications of macular recovery function, evaluated by means of nyctometry, and the retinal situation in diabetics without or with early diabetic retinopathy.

Institute of Ophthalmology, Retinal Vascular Clinic, University of Padova, Padova, Italy

E Midena

T Segato

M Giuliano

Department of

Biostatistics and

Epidemiology, Fidia

Research Laboratories,

Abano Terme, Italy

M Zucchetto

Correspondence to: E Midena, MD, Institute of Ophthalmology-Policlinico, Via Giustiniani 2, 35128 Padova, Italy.

Accepted for publication 8 September 1989

\section{Patients and methods}

The patients consisted of 234 diabetics seen at the Retinal Vascular Clinic, Institute of Ophthalmology, the University of Padova. The mean duration of diabetes was 13 years (SD 7). All patients had a visual acuity of $1 \cdot 0$ (equivalent to $6 / 6$ ). Their retinopathy ranged from no retinopathy (99 subjects) to early background retinopathy. The retina was examined by direct and indirect ophthalmoscopy and fundus biomicroscopy. Fluorescein angiography was performed in diabetics clinically classified without retinopathy; it was negative in all these subjects. The patients with retinopathy were divided into two groups: I, with some microaneurysms and some retinal haemorrhages, but total haemorrhages less than standard photograph 2A of the Early Treatment Diabetic Retinopathy Study
(ETDRS) protocol (83 subjects); II, with group I lesions and some hard exudates, less than standard photograph 3 of the ETDRS protocol, all located outside the macular zone (52 patients).${ }^{8}$ No patient had soft exudates, intraretinal microvascular abnormalities, or signs of macular oedema, nor had any patient previously received photocoagulation. No cardiovascular, neurological, or other ocular disease was present at the time of the examination. Among these diabetics 134 randomly selected subjects underwent blood sugar determination (Reflocheck, Boehringer, Mannheim, FRG) immediately after the macular recovery function had been studied.

\section{NYCTOMETRY}

Nyctometry is a dynamic measure of the initial 2minute course of macular recovery function following preadaptation to a strong uniform illumination of a large area of the retina. The instrument we use is the Registriert Nyktometer (Carl Zeiss, Jena, DDR) which allows a minimal intervention by the examinator. Nyctometry was performed on both eyes; the recording on a single eye took 6.5 minutes.

The patient looks into a white coloured globe, in which a sight test chart is placed opposite to the patient's eye. The test chart is composed of 10 lines (visual acuity from 0.1 to 1.0 ) of numbers, which can be modified to prevent memorisation. The optical system of the instrument is made to determine long distance visual acuity.

Briefly, nyctometry consists of three steps. The first is a 3-minute adaptation to strong light $\left(7000 \mathrm{asb} ; 2200 \mathrm{~cd} / \mathrm{m}^{2}\right)$. The light is then switched off and the 2-minute initial phase of dark adaptation begins. During this phase the patient has to read the test signs (illumination $0.5 \mathrm{asb} ; 0.16 \mathrm{~cd} /$ $\mathrm{m}^{2}$ ); thus we record the increase in visual acuity as a function of time. The last step is a measure of patient's sensitivity to glare (for details of technique see Frost-Larsen et $\left.a l^{9}\right)$. The last two phases are graphically recorded on a form. The individual graphic is compared with standard age-related ranges of normality $(20-30,31-50$, $51-60,61-70$ years). The result is reported as reduced or normal. Reduced nyctometry means a value below the 2.5 percentile of the normal age-related range.

The statistical analysis was by the $\chi^{2}$ test. When the results in the two eyes were similar, data from the right eye are reported. In addition the results of nyctometry were quantified by the summation method proposed by Frost-Larsen and Larsen. ${ }^{4}$ This method, called by the proposers 'intensified calculation of macular recovery time', gives an absolute number for 
TABLE I Nyctometry determinations compared with retinal state of diabetic patients

\begin{tabular}{lllll}
\hline & & \multicolumn{2}{l}{ Retinopathy } & \\
\cline { 3 - 4 } Nyctometry & No retinopathy & $I$ & II & Total \\
\hline Reduced & $37(37 \cdot 4 \%)$ & $38(45 \cdot 8 \%)$ & $35(67 \cdot 3 \%)$ & 110 \\
Normal & $62(62 \cdot 6 \%)$ & $45(54 \cdot 2 \%)$ & $17(32 \cdot 7 \%)$ & 124 \\
Total & 99 & 83 & 52 & 234 \\
\hline $\mathrm{p}=0.002$ & & &
\end{tabular}

each examination. This number results from the sum of the products of ten times each reached step of visual acuity $(0 \cdot 1=1,0 \cdot 2=2 \ldots)$ and the difference between 120 seconds (the whole 2minute course of cone adaptation) and the actual time (in seconds) taken to reach that step.

\section{Results}

In a preliminary phase we performed nyctometry in a large population of normal subjects of different ages (20 to 70 years). Their macular recovery function always lay in the normal age related range given by the manufacturer of the instrument (Midena et al, unpublished data).

Table I summarises the results of nyctometry evaluation in our diabetic population. The proportion of abnormal (reduced) results increased with deterioration of the retinopathy: $45 \cdot 8 \%$ in group I, $67 \cdot 3 \%$ in group II. Moreover we observe that $37 \cdot 4 \%$ of the patients classified (clinically and angiographically) without retinopathy showed altered macular recovery function. The nyctometry results were significantly related $\left(\chi^{2}=12 \cdot 34, \mathrm{df}=2, \mathrm{p}=0.002\right)$ to the progression of subclinical and clinical diabetic retinopathy.

The blood glucose level at the time of nyctometry determination did not influence the result of the examination (Table II). The quantitative data of nyctometry (Table III) showed a decrease of this function with age in normal and diabetic subjects.

Moreover it showed in each age group a worsening of macular recovery function with deterioration of the retinopathy.

\section{Discussion}

Nyctometry is a standardised technique used to

TABLE II Blood glucose level at the time of nyctometry examination in 134 randomly selected diabetics

\begin{tabular}{llllll}
\hline \multirow{2}{*}{ Nyctometry } & $6 \cdot 7$ & $6 \cdot 8-8 \cdot 9$ & $9 \cdot 0-11 \cdot 0$ & $>11 \cdot 0$ & Total \\
\cline { 2 - 5 } & $6 \cdot 7$ caemia $($ mmolll $)$ & $20(58 \cdot 8 \%)$ & $20(64 \cdot 5 \%)$ & 69 \\
Reduced & $11(33 \cdot 3 \%)$ & $18(50 \cdot 0 \%)$ & $14(41 \cdot 2 \%)$ & $11(35 \cdot 5 \%)$ & 65 \\
Normal & $22(66 \cdot 7 \%)$ & $18(50 \cdot 0 \%)$ & 34 & 31 & 134 \\
Total & 33 & 36 & & \\
\hline
\end{tabular}

$\mathrm{p}=0.065$.

TABLE III Nyctometry quantified by the summation method (Frost-Larsen and Larsen ${ }^{4}$ ). In normal and diabetic subjects the absolute value of nyctometry has been calculated by the summation method (see text) and the mean of each age group is reported (standard deviation in brackets)

\begin{tabular}{lllll}
\hline \multirow{5}{*}{ Age group } & Normals & Diabetics & \\
\cline { 3 - 5 } & No retinopathy & Retinopathy I & Retinopathy II \\
\hline $10-30 \mathrm{yr}$ & $712 \cdot 5(306 \cdot 8)$ & $570 \cdot 2(446 \cdot 7)$ & $502 \cdot 0(349 \cdot 3)$ & $139 \cdot 3(102 \cdot 3)$ \\
$31-50 \mathrm{yr}$ & $697 \cdot 0(217 \cdot 8)$ & $538 \cdot 2(359 \cdot 0)$ & $305 \cdot 1(180 \cdot 3)$ & $348 \cdot 6(264 \cdot 8)$ \\
$51-60 \mathrm{yr}$ & $499 \cdot 2(200 \cdot 8)$ & $388 \cdot 2(301 \cdot 2)$ & $389 \cdot 0(308 \cdot 0)$ & $197 \cdot 5(171 \cdot 7)$ \\
$61-70 \mathrm{yr}$ & $262 \cdot 3(123 \cdot 9)$ & $332 \cdot 3(151 \cdot 3)$ & $248 \cdot 1(156 \cdot 5)$ & $206 \cdot 3(181 \cdot 5)$ \\
\hline
\end{tabular}

measure macular recovery function in a short time. The test lasts 6.5 minutes only. The result is easily compared with standard age related curves. The normal data from our laboratory appeared to be identical to those given by the manufacturer; we therefore use the age ranges provided by the manufacturer. In consequence the result is independent of the patient's age. This is a great advantage in respect of other functional methods, where normative age related data are not available. Some authors consider nyctometry to be a technique for measuring dark adaptation - more precisely, the dark adaptation of the cone system. ${ }^{10}$ Nyctometry quantifies not only the dark adaptation of the cone system but also the macular sensitivity to glare. We therefore think it is entirely adequate for studying the function of the macula.

Previous reports on nyctometry have suggested that it can be used to predict the progression of background retinopathy to the proliferative form. ${ }^{249}$ Similar results have been reported with the electroretinogram oscillatory potentials. ${ }^{23}$

In contrast very few reports have dealt with the use of nyctometry in diabetics without or with early retinopathy. Gliem and Schultze ${ }^{11}$ showed that 42 of 159 eyes of diabetics without retinopathy gave abnormal nyctometry results. But their study suffers from some methodological inaccuracies: they did not use age related normal ranges; the results of nyctometry were related to nyctometry without considering the age of the patient. Frost-Larsen and Larsen ${ }^{4}$ reported that two out of 57 eyes of diabetics without retinopathy gave reduced nyctometry results, but their paper does not consider this problem in detail.

Our data clearly show, in a well defined series of patients, that reduced nyctometry is directly and strongly related to the progression of retinal (functional and anatomical) derangements due to diabetes. This is confirmed by a quantitative method. The limit of this and the other methods proposed to quantify nyctometry is the inadequacy of each to describe adequately the curve obtained with this test. The final visual acuity and the Frost-Larsen and Larsen summation method show too great a variation in results. ${ }^{41}$ The area under the curve - proposed by Brinchmann-Hansen et $a l^{12}$ - gives equal results with different curves.

We therefore consider that so far the best way to classify nyctometry results is qualitatively (normal, reduced). Our data are similar to those obtained by means of other functional tests. ${ }^{35}$ 13-15

Moreover the results of nyctometry are independent of the glycaemic status of a patient who is healthy at the time of the investigation. No comparable data are available from previous studies performed with other functional tests in diabetics. We have also shown that $37 \cdot 4 \%$ of subjects considered by current standards to be unaffected by retinopathy give reduced nyctometry results, an indication of abnormal macular function. At present we do not know why macular recovery function is abnormal in these subjects. They may perhaps represent, among diabetics without retinopathy, a group in whom 
retinopathy will soon appear. A similar hypothesis has been proposed by Della Sala et $a l^{13}$ using a contrast sensitivity test. To confirm (or not) this hypothesis a five-year prospective study is in progress.

Our data also suggest that nyctometry might be used as a simple, quick, and non-invasive test to monitor the function of the macula in diabetics during intensified metabolic control or drug treatment. A further implication of our results on nyctometry in diabetic patients should also be pointed out. The site of the initial phase of dark adaptation is not yet completely defined, but it is thought to involve neural processes in the retina. ${ }^{16-18}$ Nyctometry is thus a test which provides information about the function of a specific part not only of the visual system but also of the retina. This could provide new insight into the pathophysiology of diabetic retinopathy.

1 Bresnick GH. Diabetic retinopathy viewed as a neurosensory disorder. Arch Ophthalmol 1986; 104: 989-90.

2 Simonsen SE. The value of oscillatory potential in selecting juvenile diabetics at risk of developing proliferative retinopathy. Acta Ophthalmol (Kbh) 1980; 58: 865-78.

3 Bresnick GH, Korth K, Groo A, Palta M. Electroretinographic oscillatory potentials predict progression of diabetic retinooscillatory potentials predict progression of
pathy. Arch Ophthalmol $1984 ; 102$ : 1307-11.

4 Frost-Larsen K, Larsen HW. Macular recovery time recorded by nyctometry - a screening method for selection of patients by nyctometry - a screening method for selection of patients who are at risk of developing proliferative diabetic
pathy. Arch Ophthalmol 1985; 63 (suppl 173): 39-47.

5 Roy MS, Gunkel RD, Podgor MJ. Color vision defects in early diabetic retinopathy. Arch Ophthalmol 1986; 104· 225-8.
6 Ghafour IM, Foulds WS, Allan D, McClure E. Contrast sensitivity in diabetic subjects with and without retinopathy. Br F Ophthalmol 1982; 66: 492-5.

7 Sokol S, Moskowitz A, Skarf B, Evans R, Molitah M, Senior $B$. Contrast sensitivity in diabetic subjects with and without retinopathy. Arch Ophthalmol 1985; 103: 51-4.

8 Early Treatment Diabetic Retinopathy Study: Coordinating Centre. Manual of operations. Baltimore: Diabetic Retinopathy Coordinating Center, 1980: chapter 11.

9 Frost-Larsen K, Larsen HW, Simonsen SE. Oscillary potentials and nyctometry in insulin-dependent diabetics. Acto Ophthalmol $(K b h)$ 1980; 58: 879-88.

10 Bresnick GH. Methods to quantify diabetic retinopathy: structural and functional. In: Andreani D, Crepaldi G, Di Mario U, Pozza G, eds. Diabetic complications: early diagnosis and treatment. Chichester: Wiley, 1987: 77-91.

11 Gliem $\mathrm{H}$, Schulze DP. Sofortadaptation, Bleudungsempfindlickheit und diabetische Retinopathie. Klin Monatsbl Augenheilkd 1966; 166: 766-9.

12 Brinchmann-Hansen O, Dahl-Jorgensen K, Hanssen KS, Sandvik L. Oscillatory potentials, macular recovery time and diabetic retinopathy through 3 years of intensified insulin treatment. Ophthalmology 1988; 95: 1358-66.

13 Della Sala S, Bertoni G, Somazzi L, Stubbe F, Wilkins AJ Impaired contrast sensitivity in diabetic patients with and without retinopathy: a new technique for rapid assessment BrF Ophthalmol 1985; 69: 136-42.

14 Arden GB, Hamilton AMP, Wilson-Holt I, Ryon S, Yudkin IS, Kurtz A. Pattern electroretinograms become abnorma when background diabetic retinopathy deteriorates to proliferative stage: possible use as a screening test. $\mathrm{Br} f$ Ophthalmol 1986; 70: 330-5.

15 Eisner AE, Burns SA, Lobes LA JR, Doft BH. Cone photopigment bleaching abnormalities in diabetes. Invest Ophthalmol Vis Sci 1987; 28: 718-24.

16 Dowling JE. The site of visual adaptation. Science 1967; 155: 273-9

17 Barlow HB. Dark and light adaptation: psychophysics. In: Jameson D, Hurvich L, eds. Handbook of sensory physiology. Jameson D, Hurvich L, eds. Handbook

18 Hagerstrom-Partnoy G, Adam AJ, Brown B, Jomepolsky A Dynamics of visual adaptation are altered in vascular Dynamics of visual adaptation are altered in vascular
diseases. In: Breinin GM, Seigel IM, eds. Advances in diagnostic visual optics. Berlin: Springer, 1983: 225-31. 